\title{
International workshop on \\ Authoring Simulation and Game-Based Intelligent Tutoring
}

\author{
Paula J. Durlach* \\ U.S Army Research Institute \\ Paula.Durlach@us.army.mil
}

Summary. The use of scenario-based simulations and serious games for training has been well-accepted in many domains. Simulations require active processing and provide intrinsic feedback in an environment in which it is safe to make mistakes; however, reaping training benefits from this kind of training is often highly dependent on support from human instructors who select training scenarios, observe trainee behavior, and provide feedback, prompts, and reflective discussion. Applying the techniques of intelligent tutoring to simulation-based training could reduce reliance on human instructors. Schatz, Bowers, and Nicholson (2009) refer to this integration of intelligent tutoring strategies with simulation-based training as "advanced situated tutors." Advanced situated tutors include student models whose data are used to apply adaptive instructional strategies to selection of simulation events, instructional content, and instructional support.

While a small collection of advanced situated tutors exist, similar to standard intelligent tutors, creation of these systems requires a wide range of expertise and substantial resources. Many organizations that use traditional multimedia training for their personnel recognize the potential benefits of adding simulation-based or gamebased elements to training, and would readily accept advanced situated tutors were it not for the high upfront costs the creation of these systems currently entail. Authoring tools for advanced situated tutors could facilitate the development process and reduce cost. This workshop will help characterize the current state of the art and identify outstanding issues and future potential approaches to meeting this objective. The workshop will be a combination of presentations and discussion.

Organizing Committee: Antonija Mitrovic (Univ. of Canterbury), Stephen Gilbert (Iowa State University), Stephen Blessing (University of Tampa).

Review Committee: Brandt Dargue (Boeing Research and Technology), Lewis Johnson, (Alelo Inc.), Allen Munro (University of Southern California), Robert Sottilare (Army Research Laboratory), Alicia Sage (Alelo, Inc.), Randy Spain (U.S. Army Research Institute), Bruce Perrin (Boeing Research and Technology), Chas Murray (Carnegie Learning), Brendon Towle (Carnegie Learning), Glenn Martin (I. for Simulation and Training, Univ. of Central Florida).

* Workshop Chair. 